\title{
Long-Term Treatment of Rats with Haloperidol: Lack of an Effect on Brain N-Acetyl Aspartate Levels
}

\author{
Juan Bustillo*, 1,2, Ranee Barrow', Rodrigo Paz ${ }^{2,5,6}$, Jennifer Tang ${ }^{3}$, Navid Seraji-Bozorgzad ${ }^{3}$, \\ Gregory J Moore ', Federico Bolognani ${ }^{2}$, John Lauriello', Nora Perrone-Bizzozero' ${ }^{2}$ and Matthew P Galloway ${ }^{3}$ \\ 'Department of Psychiatry, University of New Mexico Health Sciences Center, Albuquerque, NM, USA; ${ }^{2}$ Department of Neurosciences, University \\ of New Mexico Health Sciences Center, Albuquerque, NM, USA; ${ }^{3}$ Department of Psychiatry and Behavioral Neurosciences, Wayne State \\ University School of Medicine, Detroit, MI, USA; ${ }^{4}$ Department of Psychiatry and Radiology, Penn State University College of Medicine, Hershey, \\ PA, USA; ${ }^{5}$ Departamento de Psiquiatria y Neurociencias, Universidad Diego Portales, Santiago, Chile; ${ }^{6}$ Instituto Psiquiatrico Dr Jose Horwitz \\ Barak, Universidad Diego Portales, Santiago, Chile
}

\begin{abstract}
Proton magnetic resonance spectroscopy ( $\mathrm{H}$-MRS) studies of schizophrenia suggest an effect of the disease or of antipsychotic medications on brain $\mathrm{N}$-acetyl aspartate (NAA), a marker of neuronal viability. We studied in rat the effect of haloperidol on NAA, glutamate, and glutamine in several brain regions where metabolite reductions have been reported in chronically medicated patients with schizophrenia. Two groups of 16 rats each were treated with haloperidol depo (38 mg/ $/ \mathrm{kg} / \mathrm{month}$ ) and vehicle for 6 months and were killed. Concentrations of metabolites were determined by high-resolution magic angle proton magnetic resonance spectroscopy (HRMAS 'H-MRS) at II.7T in ex-vivo punch biopsies from the following brain regions: medial frontal and cingulate cortex, striatum, nucleus accumbens, dorsal and ventral hippocampus, amygdala, and temporal cortex. Factorial ANOVA of NAA concentrations revealed no significant effect of drug group $(F(1,2 \mid 2)=1.5 ; p=0.22)$ or a group by brain region interaction $(F(7,2 \mid 2)=1.0 ; p=0.43)$. There was a significant main effect of region $(F(7,212)=17.8 ; p<0.00$ I) with lower NAA in the striatum. A prolonged exposure to the dopamine D2 receptor blockade effects of haloperidol does not result in changes in NAA, glutamate, glutamine, and other metabolites in the proton spectrum. These results are consistent with the only other two studies of the effect of antipsychotic drugs on NAA in the rat brain. The documented lower NAA in chronically treated schizophrenia patients is most likely not a simple effect of antipsychotic medications. Neuropsychopharmacology (2006) 31, 75I-756. doi:I0.1038/sj.npp. I300874; published online 3I August 2005
\end{abstract}

Keywords: N-acetyl aspartate; glutamate; haloperidol; antipsychotic; schizophrenia; H-MRS

\section{INTRODUCTION}

Proton magnetic resonance spectroscopy $\left({ }^{1} \mathrm{H}\right.$-MRS) studies of schizophrenia have reported reduced $\mathrm{N}$-acetyl aspartate (NAA) mainly in frontal and mesial temporal regions, mostly in chronically treated patients (for a review, see Rowland et al, 2001). NAA is produced in the neuronal mitochondria and is found almost exclusively in the neuron (Tsai and Coyle, 1995; Moffet and Namdoori, 1995). Although initially considered a relatively stable marker of neuronal density, NAA is now also viewed as a sensitive marker of neuronal viability and function (Moore and Galloway, 2002). We have presented preliminary long-

\footnotetext{
*Correspondence: Dr J Bustillo, Department of Psychiatry MSC09 5030, University of New Mexico, Research Division 2400 Tucker NE, Albuquerque, NM 87|31-000I, USA, Tel: + I 505272 558I, Fax: + | 505272 5572, E-mail: jbustillo@salud.unm.edu

Received 8 March 2005; revised 23 June 2005; accepted I2 July 2005 Online publication: 18 July 2005 at http://www.acnp.org/citations/ Npp07| 805050 | 62/default.pdf
}

itudinal data in schizophrenia patients with history of minimal previous treatment that documents reductions in frontal NAA during the first year of treatment with antipsychotic medications (Bustillo et al, 2002). Antipsychotic drugs exert both their therapeutic and deleterious extrapyramidal side effects by blocking dopamine D2 receptors and have been shown to lead to both structural and metabolic changes in the brain (Keshavan et al, 1994; Chakos et al, 1995; Holcomb et al, 1996). Hence, it is possible that NAA reductions in schizophrenia are the result of antipsychotic medications, but separating the impact of the disease from the treatment is problematic in clinical populations. The two studies that assessed the effect of exposure to antipsychotic agents on NAA measures in rat brain found negative results (Lindquist et al (2000), 1 week and Bustillo et al (2004), 6 week exposure). However, a more prolonged exposure that better corresponds to the longer term treatments typical in clinical populations may still induce reductions in NAA. The current study investigated the effects of 6 months of haloperidol, an 
antipsychotic with high D2 affinity, on NAA and other metabolite levels on various brain regions in rats, using high-resolution magic angle spin (HR-MAS) ${ }^{1} \mathrm{H}$-MRS.

\section{METHODS}

\section{Drug Exposure}

All animal experiments were approved by the Institutional Animal Care and Use Committee of the University of New Mexico (UNM) Health Sciences Center and were performed according to the guidelines of the National Institutes of Health. Male Sprague-Dawley rats weighing about $300 \mathrm{~g}$ each were purchased from Harlan (Indianapolis, IN), housed in pairs and allowed to acclimatize for 2 weeks before the start of the study. Rats were housed at the UNM animal facility under a 12:12 light: dark cycle (lights on at $7 \mathrm{am})$ and received Purina rat chow and tap water ad libitum. After a handling period of about 1 week, rats were administered by intramuscular (i.m.) injection $38 \mathrm{mg} / \mathrm{kg} /$ month of haloperidol-depo ( $n=16$; Novaplus). This dose is in the high range of similar rat studies of chronic exposure (about 6 months) of haloperidol-depo (Andreassen and Jorgensen (1995) and Meshul et al (1996) used $38 \mathrm{mg} / \mathrm{kg} /$ month; See et al (1992) and Grimm et al (1998), used $21 \mathrm{mg} /$ $\mathrm{kg} / \mathrm{Q} 3$ weeks). Rats were lightly anesthetized with isoflurane and half of the calculated drug volume was injected in each hind leg. Dosages were given once per month (between 1 and $4 \mathrm{pm})$ for 6 months. Control rats $(n=16)$ received equivalent volumes of purified sesame oil i.m. following the same procedures used with the haloperidol-depo group.

At 1 month after administration of the sixth injection, rats were exposed briefly to isoflurane and then killed by decapitation. Brains were rapidly removed, placed into rat brain matrix and $2 \mathrm{~mm}$ coronal slices obtained on an icechilled stage. Four slices were selected which approximately corresponded to the following sections of a standard rat brain atlas (Paxinos and Watson (1998); + or - refers to anterior or posterior from Bregma, respectively): slice 1, +3.7 to $+1.7 \mathrm{~mm}$; slice $2,+1.7$ to $-0.3 \mathrm{~mm}$; slice $3,-2.3$ to $-4.3 \mathrm{~mm}$; and slice 4 from -4.3 to -6.3 . Regions of interest were identified visually in each slice (Galloway et al, 1986): medial prefrontal cortex-MF (slice 1), cingulate cortex-Ci, anterior striatum-Str and accumbens nuclei-Acc (slice 2), dorsal hippocampus-HiD and amygdale-Amy (slice 3), and ventral hippocampus-HiV and auditory cortex-Au (slice 4). These regions were selected because of their distinct dopamine projection fields and/or their homologous overlap with areas studied with clinical ${ }^{1} \mathrm{H}$-MRS in chronically treated schizophrenia subjects (Rowland et al, 2001). Two millimiter circular punches were obtained from appropriate regions and immediately placed in precooled plastic centrifuge tubes and frozen on dry ice. Samples were stored at $-80^{\circ} \mathrm{C}$ until HR-MAS ${ }^{1} \mathrm{H}-\mathrm{MRS}$ analysis.

\section{HR-MAS ${ }^{1} \mathrm{H}$-MRS}

Samples $(\sim 4 \mathrm{mg})$ were weighed and then inserted into a $10 \mu \mathrm{l}$ zirconium rotor (Bruker) containing $4 \mu \mathrm{l} \mathrm{PO}_{4}$ buffer ( $\mathrm{pH}$ 7.4) containing $50 \% \mathrm{D}_{2} \mathrm{O}$, Na-formate for autophasing $(8.44 \mathrm{ppm})$, and trimethylsilane as reference $(0 \mathrm{ppm})$. Rotors, maintained at $4^{\circ} \mathrm{C}$, were spun at $4.2 \mathrm{kHz}$ at $54.7^{\circ}$ relative to Bo (static magnetic field) in a Bruker Avance $11.7 \mathrm{~T} 500 \mathrm{MHz}$ magnet using a rotor-synchronized CPMG (Cheng et al, 1997) pulse sequence with $\mathrm{TR}=3500 \mathrm{~ms}$, bandwidth $8 \mathrm{kHz}, 16 \mathrm{k}$ complex points analyzed, 256 averages, and Taq $15 \mathrm{~min}$. Spectra were analyzed with a custom LCModel (Provencher, 1993) and absolute quantities of each neurochemical determined, weight corrected, and expressed as $\mathrm{nmol} / \mathrm{mg}$ wet weight (see Figure 1). The precision of the LCModel fit to the spectral data was estimated with Cramer Rao lower bounds, which were typically $<10 \%$; values $>20 \%$ were not considered for further analysis. The LC model was constructed with a basis set of 27 neurochemicals.

Based on the clinical schizophrenia literature (Rowland et al, 2001), we hypothesized that NAA would be reduced in frontal and temporal regions with exposure to haloperidol. An ancillary hypothesis was that glutamine in the cingulate cortex would be decreased by treatment with haloperidol (based on Theberge et al, 2003). Each metabolite level was analyzed with a 2 (treatment group) by 8 (region) factorial ANOVA using SAS software. Significant effects were followed up with Fisher's least significant difference method of post hoc comparisons. Owing to potential correlations of regional metabolite values within subjects, results were confirmed with mixed model ANOVA. Data are presented as mean $\pm S D$ in all cases.

\section{RESULTS}

The quantification of NAA, Glu, Gln, Cho, and Cre total concentrations in the eight regions of interest from haloperidol- and vehicle-treated rat groups is shown in the Table 1. For NAA, the principal metabolite of interest, factorial ANOVA revealed no significant group by region interaction $(\mathrm{F}(7,212)=1.0, p=0.43)$ and no main effect of group $(\mathrm{F}(1,212)=1.5, p=0.22)$. These results do not support an effect of antipsychotic drug exposure on NAA levels in any of the regions studied. However, there was a significant main effect of region $(\mathrm{F}(7,212)=17.8, p<0.001)$. Post hoc pairwise comparisons by $t$-test revealed significantly different NAA $(p<0.05)$ in the following areas: auditory cortex higher than all others; dorsal hippocampus, cingulate and mediofrontal cortex higher than the remaining regions; and ventral hippocampus, amygdala, and accumbens higher than striatum, which was the region with the lowest NAA level. These higher concentrations in cortex and lowest in striatum are consistent with the previous rat studies (Koller et al, 1984; Florian et al, 1996, Bustillo et al, 2004). Factorial ANOVAs also failed to document a drug main effect or interaction $(p>0.05)$ for the other metabolites measured: alanine, aspartate, betaine, PEA, choline, glutamine, GPC, lactate, NAAG, succinate, taurine, GABA, glycine, glutamate, GSH, inositol, phospho choline, and creatinine total. Finally, the results did not significantly change with mixed model ANOVA reanalyses.

\section{DISCUSSION}

In rats exposed to haloperidol for 6 months we found no differences in NAA, Glu, Gln, Cho, or Cre concentrations in medial frontal, cingulate and auditory cortices, striatum, 


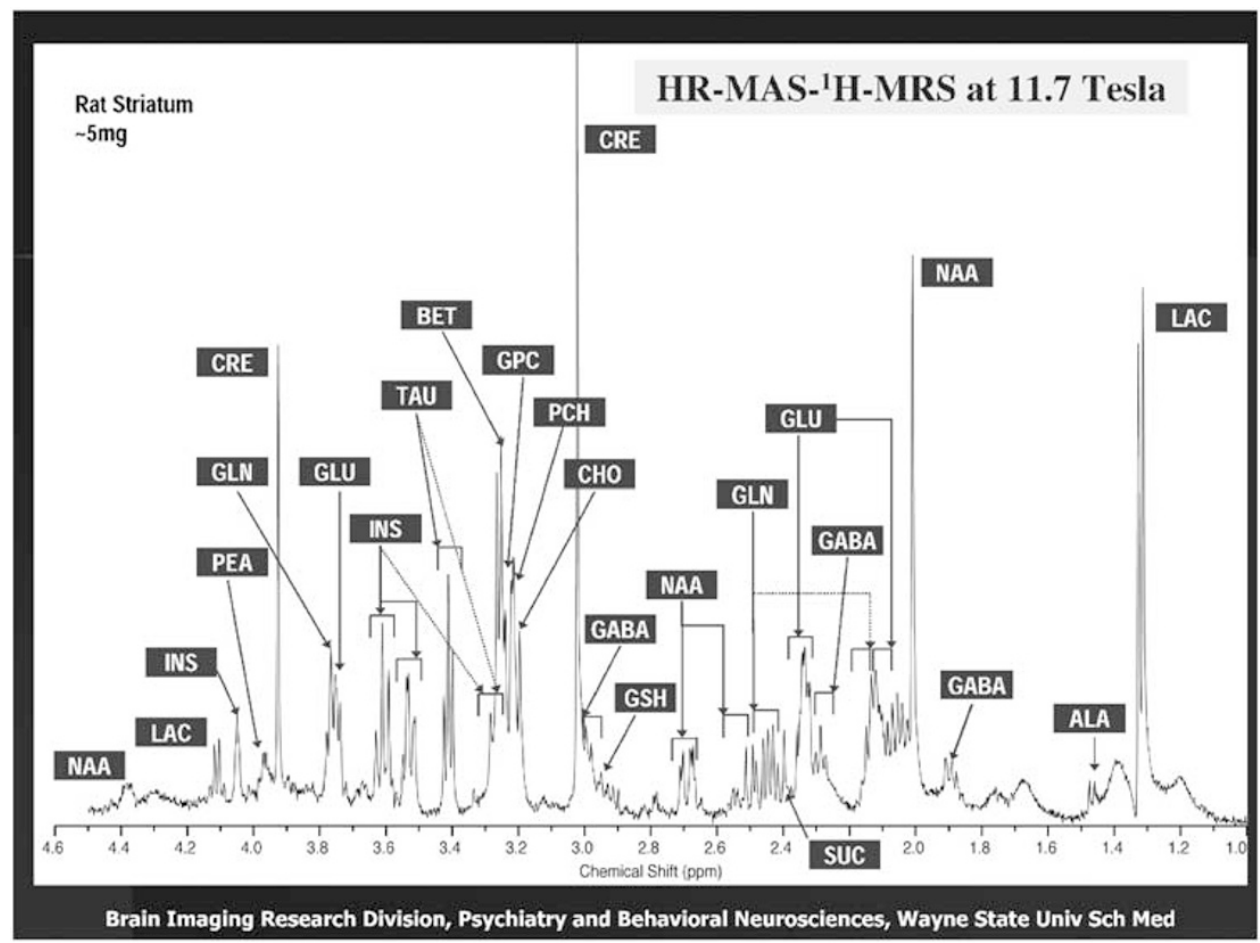

Figure I Example of a representative 'H-MRS spectrum from an intact $5 \mathrm{mg}$ sample of rat striatum acquired with HR-MAS at I I.7T. NAA, N-acetyl aspartate; LAC, lactate; INS, inositol; PEA, phosphorylethanolamine; CRE, creatine; GLU, glucose; TAU, taurine; BET, betaine; GPC, glycerophosphorylcholine; PHC, phosphocholine; $\mathrm{CHO}$, choline; GABA, gamma amino butyric acid; GSH, gluthathione; GLN, glutamine; SUC, succinate, and ALA, alanine.

accumbens, amygdala, dorsal or ventral hippocampus. These neurometabolites are of interest because they are often reported in clinical ${ }^{1} \mathrm{H}$-MRS studies of schizophrenia. We are aware of only two previous studies assessing the impact of antipsychotic drugs on NAA brain levels in animals. Lindquist et al (2000) treated rats with intraperitoneal daily injections of olanzapine $(1 \mathrm{mg} / \mathrm{kg} / \mathrm{day} ; n=8)$, clozapine $(10 \mathrm{mg} / \mathrm{kg} /$ day; $n=8)$, haloperidol $(0.2 \mathrm{mg} / \mathrm{kg} /$ day; $n=8)$, and vehicle $(n=8)$ for 1 week. Ratios of NAA to creatine (NAA/Cre) were calculated from spectra measured in vivo at $4.7 \mathrm{~T}$ with single voxel ${ }^{1} \mathrm{H}$-MRS, before, after 1 day, and at the end of treatment. The voxel studied was $0.216 \mathrm{cc}$ and located '... in front of the cerebellum, and within the volume defined by the left and right ventricle' (Lindquist et al, 2000). There were no differences in NAA/ Cre between the four groups at the end of treatment. There was a small reduction in NAA/Cre between baseline and 1 week values only in the olanzapine-treated group $(1.16 \mathrm{vs}$ $1.12 ; p<0.04)$. However, this was not a predicted result, uncorrected for multiple comparisons and the authors interpreted the overall findings as not supportive of a medication effect on NAA/Cre ratios.

We studied three groups of eight rats treated with haloperidol $(6 \mathrm{mg} / \mathrm{kg} /$ day $)$, clozapine $(70 \mathrm{mg} / \mathrm{kg} /$ day $)$, and vehicle for 6 weeks using gastric gavage twice daily (Bustillo et al, 2004). Animals were killed and concentrations of NAA were determined by high-performance liquid chromatography (HPLC) from the following brain regions: cortex, striatum, thalamus, hippocampus, and cerebellum. There were no differences in any brain region between the three groups $(\mathrm{F}(2,24)=0.034 ; p=0.966)$. These three studies, examining acute, intermediate, and long-term antipsychotic (typical and atypical) exposure, with different methods (HPLC, in vivo and ex vivo ${ }^{1} \mathrm{H}$-MRS) consistently suggest an absence of drug effect on NAA in rats.

Most studies using ${ }^{1} \mathrm{H}$-MRS to study NAA in schizophrenia involve cross-sectional assessments of patients chronically treated with mainly typical antipsychotics. These studies report lower NAA or NAA/Cre in several brain structures like mesial temporal lobe, anterior cingulate cortex; dorsolateral prefrontal cortex (DLPFC), larger $(8-12 \mathrm{cc})$ prefrontal locations; thalamus, and the cerebellum, but there are inconsistencies and several negative studies (for a review, see Rowland et al, 2001).

Few longitudinal studies of schizophrenia have assessed NAA in the context of antipsychotic drug treatment. Choe et al (1996) found no changes in left frontal NAA/Cre during naturalistic treatment with typical and atypical agents. Bertolino et al (2001), in a retrospective study, reported higher NAA/Cre in the DLPFC in patients while treated with antipsychotic medication compared to when they were medication free. We found reductions in left frontal NAA in patients with minimal prior lifetime antipsychotic exposure (less than 3 weeks) during the first year of randomized treatment with either haloperidol or quetiapine (Bustillo et al, 2002). Inconsistent findings in these longitudinal studies likely reflect methodological differences, but our preliminary results (Bustillo et al, 2002) suggest frontal NAA reductions early in schizophrenia in the context of initial antipsychotic exposure. Results from the present study do not support a simple medication effect. Progression of the disease or an interaction between disease and antipsychotic medications are alternative explanations. 
Table I Metabolic Profile of MR-Visible Compounds (nmol/mg Wet Weight) from Eight Brain Regions in Rats Exposed to Haloperidol and Vehicle Treatments for 6 Months (Means \pm Standard Deviations)

\begin{tabular}{|c|c|c|c|c|c|c|c|c|}
\hline & Medial frontal & Cingulate cortex & Striatum & Accumbens & Hippocampus dorsal & Amygdala & Hippocampus ventral & Auditory cortex \\
\hline \multicolumn{9}{|l|}{ NAA } \\
\hline Haloperidol & $5.68 \pm 1.13(N=13)$ & $6.07 \pm 0.98(N=15)$ & $4.37 \pm 0.96(N=16)$ & $5.09 \pm 0.60(N=15)$ & $6.10 \pm 0.55(N=15)$ & $5.05 \pm 1.00(N=16)$ & $5.05 \pm 1.1 \mid(N=11)$ & $6.10 \pm 1.36(N=12)$ \\
\hline Vehicle & $6.01 \pm 0.77(N=13)$ & $5.88 \pm 0.81(N=16)$ & $4.45 \pm 0.79(N=16)$ & $4.95 \pm 0.44(N=14)$ & $5.91 \pm 1.00(N=13)$ & $5.20 \pm 0.64(N=15)$ & $5.33 \pm 0.64(N=15)$ & $6.90 \pm 0.89(N=14)$ \\
\hline \multicolumn{9}{|l|}{ Cho } \\
\hline Haloperidol & $0.21 \pm 0.30(N=16)$ & $0.24 \pm 0.16(N=15)$ & $0.36 \pm 0.27(N=16)$ & $0.32 \pm 0.28(N=15)$ & $0.28 \pm 0.18(N=15)$ & $0.33 \pm 0.34(N=16)$ & $0.26 \pm 0.30(N=13)$ & $0.29 \pm 0.31(N=13)$ \\
\hline Vehicle & $0.22 \pm 0.22(N=15)$ & $0.24 \pm 0.15(N=16)$ & $0.27 \pm 0.09(N=15)$ & $0.36 \pm 0.27(N=14)$ & $0.41 \pm 0.38(N=13)$ & $0.31 \pm 0.40(N=15)$ & $0.31 \pm 0.25(N=15)$ & $0.40 \pm 0.44(N=15)$ \\
\hline \multicolumn{9}{|l|}{ Gln } \\
\hline Haloperidol & $1.55 \pm 1.10(N=16)$ & $1.39 \pm 0.33(N=15)$ & $2.09 \pm 0.59(N=16)$ & $2.27 \pm 0.53(N=15)$ & $1.68 \pm 0.3 \mid(N=\mid 5)$ & $2.13 \pm 0.36(N=16)$ & $1.41 \pm 0.74(N=13)$ & $2.05 \pm 0.31(N=12)$ \\
\hline Vehicle & $1.51 \pm 0.67(N=15)$ & $1.41 \pm 0.61 \quad(N=16)$ & $2.18 \pm 0.34(N=15)$ & $2.37 \pm 0.44(N=14)$ & $1.74 \pm 0.38(N=13)$ & $2.05 \pm 0.58(N=15)$ & $1.74 \pm 0.52(N=15)$ & $1.98 \pm 0.72(N=15)$ \\
\hline \multicolumn{9}{|l|}{ Glu } \\
\hline Haloperidol & $6.42 \pm 3.54(N=16)$ & $7.39 \pm 1.13(N=14)$ & $6.13 \pm 1.12(N=16)$ & $6.01 \pm 1.06(N=14)$ & $7.34 \pm 0.60(N=15)$ & $6.76 \pm 1.35(N=16)$ & $5.81 \pm 2.89(N=13)$ & $7.30 \pm 2.64(N=13)$ \\
\hline Vehicle & $7.51 \pm 2.86(N=15)$ & $7.45 \pm 1.09(N=16)$ & $6.27 \pm 0.82(N=14)$ & $6.07 \pm 0.74(N=14)$ & $7.41 \pm 1.17(N=13)$ & $6.85 \pm 0.93(N=15)$ & $7.11 \pm 0.81(N=15)$ & $7.86 \pm 2.52(N=15)$ \\
\hline \multicolumn{9}{|l|}{ Cre } \\
\hline Haloperidol & $3.93 \pm 2.47(N=16)$ & $5.03 \pm 0.83(N=15)$ & $4.60 \pm 0.84(N=16)$ & $4.82 \pm 0.70(N=15)$ & $5.87 \pm 0.5 ।(N=15)$ & $4.62 \pm 1.06(N=13)$ & $4.52 \pm 2.28(N=15)$ & $4.87 \pm 1.28(N=13)$ \\
\hline Vehicle & $4.55 \pm 1.87(N=15)$ & $4.90 \pm 0.81 \quad(N=16)$ & $4.73 \pm 0.78(N=15)$ & $4.56 \pm 0.64(N=15)$ & $5.64 \pm 0.92(N=13)$ & $4.77 \pm 0.72(N=13)$ & $5.30 \pm 0.7 \mid(N=\mid 5)$ & $5.15 \pm 1.61 \quad(N=15)$ \\
\hline
\end{tabular}

NAA, N-acetyl aspartate; Cho, choline; Gln, glutamine; Glu, glutamate; Cre, creatine total. 
More recent H-MRS studies at higher field (4T) suggest elevations of glutamine, a marker of glutamatergic neurotransmission, in anterior cingulate of never-medicated schizophrenia (Theberge et al, 2002). Furthermore, reductions of this metabolite in chronically treated patients (Theberge et al, 2003) suggest glutamatergic-related disease progression. The absence of an effect of haloperidol on cingulate glutamine supports the interpretation that these clinical findings are related to the disease and not exclusively to antipsychotic treatment.

Several limitations of the present study should be considered when interpreting our results. First, animals were exposed to a fixed dose of haloperidol which may not be equivalent to dosages of antipsychotics used in clinical populations. Since we did not measure haloperidol serum levels, drug underexposure cannot be excluded. However, drug delivery was assured by the use of long acting injections and the higher end $(38 \mathrm{mg} / \mathrm{kg} / \mathrm{month})$ of the dosages used in studies of tardive dyskinesia in rats were selected. With the same drug dosage and delivery protocol in rats, Andreassen et al (1996) found haloperidol serum levels of $16.5(\mathrm{SD}=2.25) \mathrm{nmol} / \mathrm{l}$, commensurate with clinical studies (Palao et al, 1994).

Second, the brain regions studied were rather large and a putative drug effect on NAA may be limited to more discrete areas or different regions. However, we specifically selected regions in which NAA reductions have been repeatedly described in chronically treated patients (frontal, cingulate, temporal cortex, and hippocampus).

Third, the animals studied were healthy and not representative of the fundamental neurobiological substrate that underlies schizophrenia, raising the possibility of an interaction between the disease and the therapeutic or deleterious effects of the medications.

Fourth, the negative findings could be due to a Type II error since the study had $80 \%$ power to detect an effect size of 1.0 for NAA in frontal and temporal regions. The one study we are aware off that specifically compared schizophrenics chronically treated with haloperidol $(n=16)$ and healthy controls $(n=18)$, reported a significant difference, with lower NAA in frontal lobe of patients and an effect size of 0.88 (Bustillo et al, 2001). Hence, it is possible that such an effect was missed in the present study and future investigations in animals with larger samples are warranted.

Fifth, the comparability of HR-MAS ex vivo ${ }^{1} \mathrm{H}$-MRS in rats and in vivo ${ }^{1} \mathrm{H}$-MRS in humans is an important issue. Enhanced spectral resolution, line widths, and signal-tonoise afforded by the high magnetic field and magic angle spinning provide information not currently available with in vivo MRS. For example, absolute values of GABA, glutamate, glutamine, and multiple choline-containing compounds are readily quantified in intact tissue with the present technique. Although MR-visible neurochemical concentrations are a unique measure distinct from tissue extracts or microdialysis, the fact that the present values were determined in integral tissue samples closely resembles the cellular environment of neurochemicals measured with in vivo ${ }^{1} \mathrm{H}$-MRS. For example, glucose and lactate notwithstanding, HR-MAS ${ }^{1} \mathrm{H}$-MRS values presented herein closely match those determined in the rat brain in vivo at 9.4 T (Pfeuffer et al 1999; Tkáč et al, 2003) Nonetheless, species differences exist in absolute values of MR-visible neurochemicals, most notably the paucity of taurine in human brain relative to levels in rodent brain. Concerns over postmortem dependent alterations in metabolite levels that occur despite rapid tissue extraction (eg increased lactate) are mitigated by comparison to appropriate vehicletreated controls. The mean results for NAA, Cre, Glu, and Gln in the present report $(6.0,4.5,7.6$, and $1.6, \mathrm{nmol} / \mathrm{mg}$ wet weight, respectively) are similar to the relative proportions of these metabolites in human studies: NAA is $10-50 \%$ greater than Cre (Bustillo et al, 2001), much greater than Gln (Theberge et al, 2003), and similar to Glu (Theberge et al, 2003). Levels of MR-visible choline in human studies actually represent several choline-contaning moieties in the brain, including choline, phosphorylcholine, and glycerophosphorylcholine, whereas the high spectral resolution of the present method allows measurement of choline separate from other choline-containing metabolites. Regarding regional variations in NAA and how these compare between rats and humans, a consensus remains to be established. In our previous human study (Bustillo et al, 2001), NAA was 12.7 in frontal lobe and 11.6 in striatum in healthy volunteers similar to the current rat study where cortical NAA was highest and striatal concentrations were lowest.

Finally, our design was cross-sectional and a longitudinal study may be more powerful to detect within-subject changes. Future animal studies with repeated in vivo ${ }^{1} \mathrm{H}$ MRS measures over several months would address this limitation.

In summary, we found no evidence in rodents that a prolonged exposure to haloperidol, more consistent with clinical practice, results in NAA reductions in several brain regions. These data suggest that the preliminary findings describing NAA reductions early in the course of schizophrenia (Bustillo et al, 2002) may not be entirely accounted for by treatment with antipsychotic medications.

\section{ACKNOWLEDGEMENTS}

We acknowledge Fernando Valenzuela, MD, PhD for assistance with administration of agents and Clifford Qualls, $\mathrm{PhD}$ for statistical support. The work was financially supported by (1) State of New Mexico Antipsychotic Algorithm Project Consultation Grant from New Mexico Department of Health; (2) Mental Illness Neuroscience Discovery Institute; and (3) National Institutes of Health DA-16736 (MPG), Michael J Fox Foundation, and the Joe Young Sr Research Fund from the State of Michigan.

\section{REFERENCES}

Andreassen O, Aamo TO, Jorgensen HA (1996). Inhibition by memantine of the development of persistent oral dyskinesias induced by long-term haloperidol treatment of rats. $\mathrm{Br} J$ Pharmacol 119: 751-757.

Andreassen O, Jorgensen $\mathrm{H}$ (1995). The rat model of tardive dyskinesia: relationship between vacuous chewing movements and gross motor activity during acute and long-term haloperidol treatment. Life Sci 57: 2263-2272.

Bertolino A, Callicott JH, Mattay VS, Weidenhammer KM, Rakow $\mathrm{R}$, Egan MF et al (2001). The effect of treatment with antipsychotic drugs on brain $\mathrm{N}$-acetylaspartate measures in patients with schizophrenia. Biol Psychiatry 49: 39-46. 
Bustillo J, Lauriello J, Rowland L, Jung R, Petropoulus H, Hart B et al (2001). Effects of chronic haloperidol and clozapine treatments on frontal and caudate brain neurochemistry in schizophrenia. Psychiatry Res Neuroimaging 107/3: 135-149.

Bustillo J, Lauriello J, Rowland L, Thomson L, Petropoulos H, Hammond $\mathrm{R}$ et al (2002). Longitudinal follow-up of neurochemical changes during the first year of antipsychotic treatment in schizophrenia patients with minimal previous medication exposure. Schizophr Res 58: 313-321.

Bustillo J, Wolff C, Myers-y-Gutierrez A, Dettmer T, Cooper T, Allan A et al (2004). Treatment of rats with antipsychotic drugs: lack of an effect on $N$ acetyl-aspartate levels. Schizophr Res 66: 31-39.

Chakos M, Lieberman J, Alvir J, Bilder R, Ashtari M (1995). Caudate nuclei volumes in schizophrenic patients treated with typical antipsychotics or clozapine. Lancet 345: 456-457.

Cheng LL, Ma MJ, Becerra L, Ptak T, Tracey I, Lackner A et al (1997). Quantitative neuropathology by high resolution magic angle spinning proton magnetic resonance spectroscopy. Proc Natl Acad Sci USA 94: 6408-6413.

Choe BY, Suh TS, Shinn KS, Lee CW, Lee C, Paik IH (1996). Observation of metabolic changes in chronic schizophrenia after neuroleptic treatment by in vivo hydrogen magnetic resonance spectroscopy. Invest Radiol 6: 345-352.

Florian C, Williams S, Bhakoo K, Noble M (1996). Regional and developmental variations in metabolite concentration in the rat brain and eye: a study using H-NMR spectroscopy and high performance liquid chromatography. Neurochem Res 21: 1065-1074.

Galloway MP, Wolf ME, Roth RH (1986). Regulation of dopamine synthesis in the medial prefrontal cortex is mediated by release modulating autoreceptors: studies in vivo. J Pharmacol Exp Ther 236: 689-698.

Grimm JW, Kruzich PJ, See RE (1998). Emergence of oral and locomotor activity in chronic haloperidol-treated rats following cortical N-methyl-D-aspartate stimulation. Pharmacol Biochem Behav 60: 167-173.

Holcomb HH, Cascella NG, Thake GK, Medoff DR, Dannals RF, Tamminga CA (1996). Functional sites of neuroleptic drug action in the human brain: PET/FDG studies with and without haloperidol. Am J Psychiatry 153: 41-49.

Keshavan MS, Bagwell WW, Haas GL, Sweeney JA, Schooler NR, Pettegrew JW (1994). Changes in caudate volume with neuroleptic treatment (letter). Lancet 344: 1434.

Koller KJ, Zaczek R, Coyle JT (1984). N-acetyl-aspartyl-glutamate: regional levels in rat brain and the effects of brain lesions as determined by a new HPLC method. J Neurochem 43: 1136-1142.
Lindquist DM, Hawk RM, Karson CN, Komoroski RA (2000). Effects of antipsychotic drugs on metabolite ratios in rat brain in vivo. Magn Reson Med 43: 355-358.

Meshul C, Bunker G, Mason J, Allen C, Janowsky A (1996). Effects of subchronic clozapine and haloperidol on striatal glutamatergic synapses. J Neurochem 67: 1965-1973.

Moffet JR, Namdoori MAA (1995). Differential distribution of $\mathrm{N}$-acetylapartylglutamate and $\mathrm{N}$-acetylapartate immunoreactivities in rat forebrain. J Neurocytol 24: 409-433.

Moore GJ, Galloway MP (2002). Magnetic resonance spectroscopy: neurochemistry and treatment effects in affective disorders. Psychopharmacol Bull 36: 5-23.

Palao D, Arauxo A, Brunet M, Bernardo M, Haro J, Ferrer J et al (1994). Haloperidol: therapeutic window in schizophrenia. J Clin Psychopharmacol 14: 303-310.

Paxinos G, Watson C (1998). The Rat Brain in Stereotaxic Coordinates. Academic Press: San Diego. pp 256.

Pfeuffer J, Tkac I, Provencher S, Gruetter R (1999). Toward an in-vivo neurochemical profile: quantification of 18 metabolites in short-echo time ${ }^{1} \mathrm{H}$ NMR spectra of the rat brain. J Magn Res 141: 104-120.

Provencher S (1993). Estimation of metabolite concentrations from localized in vivo proton NMR spectra. Magn Reson Med 30: 672-679.

Rowland L, Bustillo J, Lauriello J (2001). Proton magnetic resonance spectroscopy $\left({ }^{1} \mathrm{H}-\mathrm{MRS}\right)$ studies of schizophrenia. Semin Clin Neuropsychiatry 6: 121-130.

See RE, Chapman MA, Klitenick MA (1992). Chronic neuroleptic administration decreases extracellular GABA in the nucleus accumbens but not in the caudate-putamen of rats. Brain Res 588: $177-180$.

Theberge J, Al-Semaan Y, Williamson P, Menon RS, Neufeld RW, Rajakumar N et al (2003). Glutamate and glutamine in the anterior cingulate and thalamus of medicated patients with chronic schizophrenia and healthy comparison subjects measured with $4.0 \mathrm{~T}$ proton MRS. Am J Psychiatry 160: 2231-2233.

Theberge J, Bartha R, Drost DJ, Menon RS, Malla A, Takhar J et al (2002). Glutamate and glutamine measured with $4.0 \mathrm{~T}$ proton MRS in never-treated patients with schizophrenia and healthy volunteers. Am J Psychiatry 159: 1944-1946.

Tkáč I, Rao R, Georgieff M, Gruetter R (2003). Neurochemical profile of the rat brain determined by in-vivo ${ }^{1} \mathrm{H}-\mathrm{NMR}$ spectroscopy. Magn Res Med 50: 24-32.

Tsai G, Coyle JT (1995). N-acetylaspartate in neuropsychiatric disorders. Prog Neurobiol 46: 531-540. 